\title{
Vacuum gravitational collapse in nine dimensions
}

\author{
P. Bizoń, ${ }^{1}$ T. Chmaj, ${ }^{2,3}$ A. Rostworowski, ${ }^{1}$ B. G. Schmidt, ${ }^{4}$ and Z. Tabor ${ }^{5}$ \\ ${ }^{1}$ M. Smoluchowski Insitute of Physics, Jagiellonian University, Kraków, Poland \\ ${ }^{2}$ H. Niewodniczański Institute of Nuclear Physics, Polish Academy of Sciences, Kraków, Poland \\ ${ }^{3}$ Cracow University of Technology, Kraków, Poland \\ ${ }^{4}$ Max-Planck-Institut für Gravitationsphysik, Albert-Einstein-Institut, Golm, Germany \\ ${ }^{5}$ Department of Biophysics, Jagellonian University, Kraków, Poland \\ (Received 12 November 2005; published 16 December 2005)
}

\begin{abstract}
We consider the vacuum gravitational collapse for cohomogeneity-two solutions of the nine dimensional Einstein equations. Using combined numerical and analytical methods we give evidence that within this model the Schwarzschild-Tangherlini black hole is asymptotically stable. In addition, we briefly discuss the critical behavior at the threshold of black-hole formation.
\end{abstract}

DOI: 10.1103/PhysRevD.72.121502

PACS numbers: $04.50 .+\mathrm{h}$

\section{INTRODUCTION AND SETUP}

Over the past few years there has been a surge of interest in higher dimensional gravity, motivated by several reasons. The main reason comes from the new brane-world scenario in string theory according to which we live on a three dimensional surface (called a brane) in a higher dimensional spacetime [1]. In order to understand the phenomenology of this scenario and its experimental predictions, like production of black holes in the next generation of colliders, it becomes important to study solutions of Einstein's equations in more than four dimensions. Another reason, closer to our own motivation, has nothing to do with string theory and comes from general relativity itself. Viewing the dimension of a spacetime as a parameter of the theory is helpful in understanding which features of general relativity depend crucially on our world being four dimensional and which ones hold in general. Last, but not least, extra dimensions are fun.

In a recent paper [2] some of us showed that in five spacetime dimensions one can perform a consistent cohomogeneity-two symmetry reduction of the vacuum Einstein equations which - in contrast to the spherically symmetric reduction - admits time dependent asymptotically flat solutions. The key idea was to modify the standard spherically symmetric ansatz by replacing the round metric on the three-sphere with the homogeneously squashed metric, thereby breaking the $S O(4)$ isometry to $S O(3) \times U(1)$. In this way the squashing parameter becomes a dynamical degree of freedom and the Birkhoff theorem is evaded. This model provides a simple theoretical setting for studying the dynamics of gravitational collapse in vacuum, both numerically [2] and analytically [3].

As mentioned in [2], similar models can be formulated in higher $D=n+2$ dimensions as long as the corresponding sphere $S^{n}$ admits a nonround homogeneous metric, i.e. there exists a proper subgroup of the orthogonal group $S O(n+1)$ which acts transitively on $S^{n}$. According to the classification given by Besse [4], such transitive actions exist on all odd dimensional spheres. For example, the group $S U(n+1)$ acts transitively on $S^{2 n+1}$ and the group $\mathrm{Sp}(n+1)$ acts transitively on $S^{4 n+3}$. It is natural to ask whether the properties of gravitational collapse found in [2] are typical for this class of models or whether new phenomena appear in higher dimensions. A systematic analysis of this question appears hopeless in view of the fact that the number of degrees of freedom (squashing parameters) grows quickly with dimension, thus it seems useful to look at specific examples to get better understanding. In this note we consider a model with 1 degree of freedom which describes the squashing of the seven sphere. More concretely, we regard $S^{7}$ as the coset manifold $\mathrm{Sp}(2) / \mathrm{Sp}(1) \simeq S O(5) / S O(3)$, or equivalently, as the $S^{3}$ bundle over the $S^{4}$ base space with $S O(5) \times S O(3)$ invariant metric. In the past, such the squashed seven sphere has attracted a great deal of attention in the context of eleven dimensional supergravity [5].

We parametrize the metric in the following way

$$
d s^{2}=-A e^{-2 \delta} d t^{2}+A^{-1} d r^{2}+r^{2} d \Omega_{7}^{2},
$$

where $d \Omega_{7}^{2}$ is the metric on the unit squashed $S^{7}$ [6]

$$
d \Omega_{7}^{2}=\frac{1}{4} e^{3 B}\left(d \mu^{2}+e_{i}^{2}\right)+\frac{1}{4} e^{-4 B} E_{i}^{2},
$$

$B, A$, and $\delta$ are functions of $t$ and $r$,

$$
e_{i}=\frac{1}{2} \sin \mu\left(\sigma_{i}-\tilde{\sigma}_{i}\right), \quad E_{i}=\cos ^{2} \frac{\mu}{2} \sigma_{i}+\sin ^{2} \frac{\mu}{2} \tilde{\sigma}_{i},
$$

and $\sigma_{i}$ and $\tilde{\sigma}_{i}$ are two sets of left-invariant one-forms on $S U(2)$

$$
\begin{aligned}
& \sigma_{1}=\cos \psi d \theta+\sin \psi \sin \theta d \phi, \\
& \sigma_{2}=-\sin \psi d \theta+\cos \psi \sin \theta d \phi, \\
& \sigma_{3}=d \psi+\cos \theta d \phi,
\end{aligned}
$$

with $\tilde{\sigma}_{i}$ given by identical expressions in terms of $(\tilde{\psi}, \tilde{\theta}, \tilde{\phi})$. In this ansatz the $S O(8)$ isometry of the round $S^{7}$ is broken to $S O(5) \times S U(2)$. This is a special case of a more general 
ansatz for which the $S^{3}$ fibers are allowed to be squashed themselves [7].

Substituting the ansatz (1) into the vacuum Einstein equations, $R_{\alpha \beta}=0$, we get equations of motion for the functions $A(t, r), \delta(t, r)$ and $B(t, r)$ (in the following we use overdots and primes to denote $\partial_{t}$ and $\partial_{r}$, respectively)

$$
\begin{gathered}
A^{\prime}=-\frac{6 A}{r}+\frac{1}{7 r}\left(48 e^{-3 B}-12 e^{-10 B}+6 e^{4 B}\right) \\
-3 r\left(e^{2 \delta} A^{-1} \dot{B}^{2}+A B^{\prime 2}\right) \\
\dot{A}=-6 r A \dot{B} B^{\prime}, \\
\delta^{\prime}=-3 r\left(e^{2 \delta} A^{-2} \dot{B}^{2}+B^{\prime 2}\right), \\
\left(e^{\delta} A^{-1} r^{7} \dot{B}\right)^{\cdot}-\left(e^{-\delta} A r^{7} B^{\prime}\right)^{\prime} \\
+\frac{4}{7} e^{-\delta} r^{5}\left(6 e^{-3 B}-e^{4 B}-5 e^{-10 B}\right)=0 .
\end{gathered}
$$

It follows immediately from the above equations that if $B=0$ (no squashing), then $\delta=0$ and either $A=1$ (Minkowski), or $A=1-r_{h}^{6} / r^{6} \quad$ (SchwarzschildTangherlini), where $r_{h}$ is the radius of the black-hole horizon. We showed in [2] that the analogous two solutions in five dimensions play the role of generic attractors in the evolution of regular asymptotically flat initial data (small and large ones, respectively) and the transition between these two outcomes of evolution exhibits the type II discretely self-similar critical behavior. The aim of this note is to show that these properties are also present in nine dimensions which suggests that they are general features of vacuum gravitational collapse for this class of models.

\section{LINEAR STABILITY AND QUASINORMAL MODES}

Before presenting numerical evidence for the nonlinear stability of the Schwarzschild-Tangherlini solution, we want to discuss the results of linear perturbation theory. Linearizing Eqs. (5)-(8) around the SchwarzschildTangherlini solution we obtain the linear wave equation for the perturbation $\delta B(t, r)$

$$
\begin{aligned}
\ddot{\delta B}-\frac{1}{r^{7}} A_{0}\left(r^{7} A_{0} \delta B^{\prime}\right)^{\prime}+\frac{16 A_{0}}{r^{2}} \delta B & =0, \\
A_{0} & =1-\frac{1}{r^{6}},
\end{aligned}
$$

where we have used the scaling freedom to set the radius of the horizon $r_{h}=1$. Introducing the tortoise coordinate $x$ defined by $d x / d r=A_{0}^{-1}$, and substituting $\delta B(t, r)=$ $e^{-i k t} r^{-7 / 2} u(x)$ into (9) we get the Schrödinger equation on the real line $-\infty<x<\infty$

$$
\begin{aligned}
-\frac{d^{2} u}{d x^{2}}+V(r(x)) u & =k^{2} u, \\
V(r) & =\frac{1}{4}\left(1-\frac{1}{r^{6}}\right)\left(\frac{99}{r^{2}}+\frac{49}{r^{8}}\right) .
\end{aligned}
$$

This equation is a special case (corresponding to the gravitational tensor perturbation with $l=2$ ) of the master equation for general perturbations of the higher dimensional Schwarzschild-Tangherlini solutions derived independently by Gibbons and Hartnoll [8] and Ishibashi and Kodama [9]. Since the potential in (10) is everywhere positive, there are no bound states, which implies that the Schwarzschild-Tangherlini black hole is linearly stable.

We computed quasinormal modes, i.e. solutions of Eq. (10) satisfying the outgoing wave boundary conditions $u \sim e^{ \pm i k x}$ for $x \rightarrow \pm \infty$, using Leaver's method of continued fractions $[10,11]$. Substituting

$$
u(x(r))=\left(\frac{r-1}{r+1}\right)^{-i k / 6} e^{i k r} \sum_{n=0}^{\infty} a_{n}\left(\frac{r-1}{r}\right)^{n}, \quad a_{0}=1,
$$

into (10) we get a 12-term recurrence relation for the coefficients $a_{n}$. The prefactor in (11) fulfills the outgoing wave boundary conditions both at the horizon and at infinity, hence the quasinormal frequencies are given by the discrete values of $k$ for which the series in (11) converges at $r=\infty$. Using Gaussian elimination [11] we reduced the 12-term recurrence relation to a 3-term one. According to Leaver [10], the convergence of the series $\sum_{n=0}^{\infty} a_{n}$ is equivalent to a condition under which the 3 term recurrence relation has a minimal solution. Such the condition, formulated in terms of continued fractions, yields the transcendental eigenvalue equation which has to be solved numerically. The full details of this computation will be described elsewhere. Here, in order to interpret numerical results we need only to know that the fundamental quasinormal mode has the frequency $k_{0}=$ $3.4488-0.8601 i$ (in units $r_{h}^{-1}$ ). This mode is expected to dominate the process of ringdown. The higher modes are damped much faster (for example, the first overtone has the frequency $k_{1}=2.7548-2.6116 i$ ) so in practice they play no role in the dynamics.

\section{NUMERICAL RESULTS}

Using the same finite-difference code as in [2] we solved equations (5)-(8) numerically for several families of regular initial data. We found that the overall picture of dynamics of gravitational collapse looks qualitatively the same as in five dimensions, that is, we have dispersion to the Minkowski spacetime for small data and collapse to the Schwarzschild-Tangherlini black hole for large data [see Fig. 1(a)]. In the latter case we looked in more detail at the asymptotics of this process. We found that at some intermediate times the solution settling down to the 


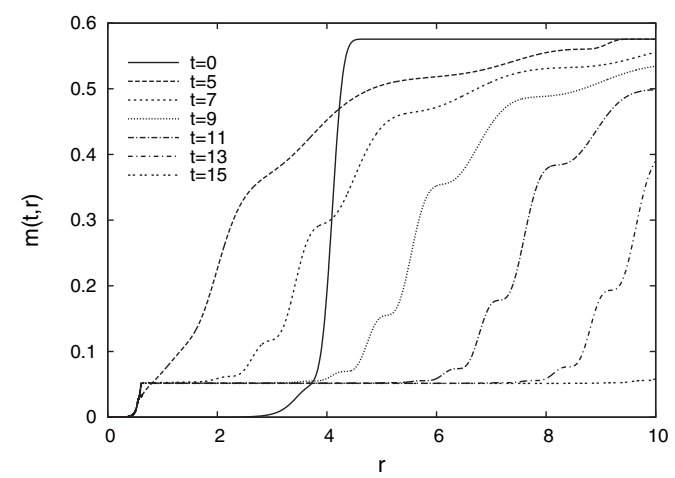

(a) Formation of a black hole

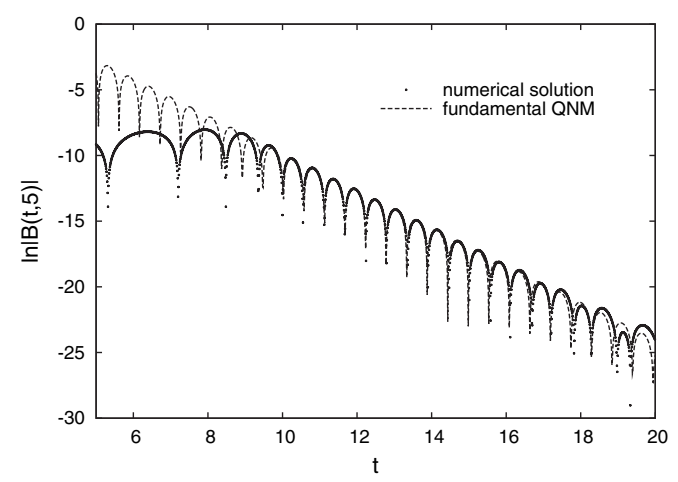

(b) Quasinormal ringing

FIG. 1. (a) For a black-hole solution we plot a series of latetime snapshots of the mass function $m(t, r)$. The initial mass function (solid line) has the form of a kink asymptoting the total mass $m(\infty)=0.575$. During the evolution the horizon develops at $r_{h}=0.606$ which corresponds to the black-hole mass $m_{B H}=$ $r_{h}^{6}=0.05$. Thus, only a small fraction of the total mass gets trapped inside the horizon while the remaining mass is being radiated away to spatial infinity, as is clearly seen from the plot. (b) We plot the time series $\ln \left|B\left(t, r_{0}\right)\right|$ at $r_{0}=5$ for the same solution as in (a), and superimpose the fundamental quasinormal mode with frequency $k_{0}=(3.4488-0.8601 i) / r_{h}$. On the time interval $10<t<16$ we get very good agreement between these two curves which confirms a well-known fact that quasinormal modes encode an intermediate time behavior of solutions at a fixed point in space [14].

Schwarzschild-Tangherlini black hole is well approximated outside the horizon by the least damped quasinormal mode. This is shown in Fig. 1(b).

At the threshold for black-hole formation we observe the type II discretely self-similar critical behavior with the echoing period $\Delta \approx 0.78$ and the black-hole mass scaling law with the universal exponent $\gamma \approx 1.64$. This is shown in Fig. 2. Note that in eight space dimensions, mass has the dimension of length ${ }^{6}$, hence $\gamma=6 / \lambda$, where $\lambda$ is the eigenvalue of the growing mode of the critical solution. Strangely enough, the product $\Delta \lambda \approx 2.85$ is approximately the same (up to numerical errors) as in five dimensions [2]. We do not know whether there is any deeper meaning behind this numerical coincidence.

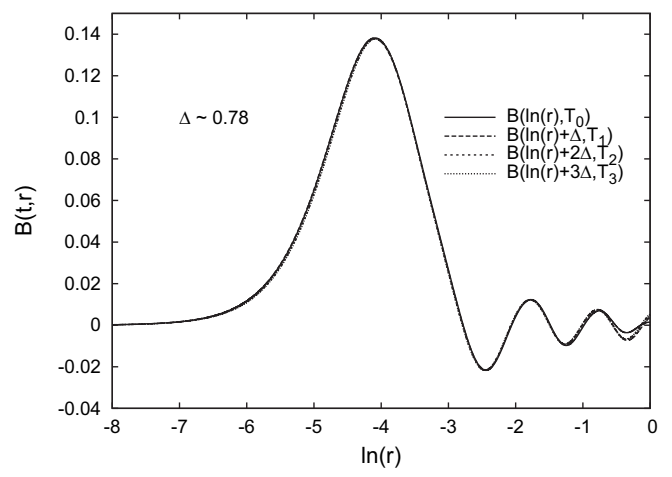

(a) Discrete self-similarity

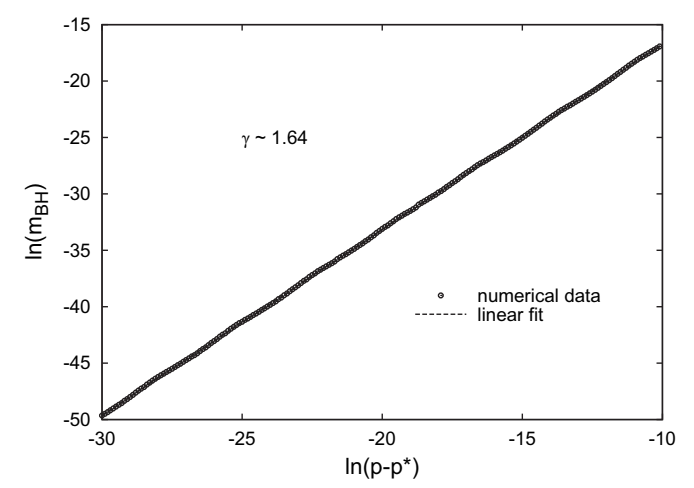

(b) Black-hole mass scaling

FIG. 2. (a) For a near-critical evolution we plot the function $B$ at some late central proper time $T_{0}$ and superimpose the next three echoes (at times $T_{n}$ ) shifted by $\ln (r) \rightarrow \ln (r)+n \Delta$. Minimization of the discrepancy between the profiles yields $\Delta \approx$ 0.78. (b) For supercritical solutions the logarithm of black-hole mass $m_{B H}$ is plotted versus the logarithmic distance to criticality. A fit of the power law $\ln \left(m_{B H}\right)=\gamma \ln \left(p-p^{*}\right)+$ const. yields $\gamma \approx 1.64$. The small wiggles around the linear fit are the imprints of discrete self-similarity; their period is equal to 2.87 in agreement with the theoretical prediction $6 \Delta / \gamma$.

\section{FINAL REMARKS}

In this note we have focused on similarities between the models in five and nine dimensions, however at the end we would like to mention two interesting qualitative differences which in our opinion are worth investigation.

The first difference is the existence of the second static solution corresponding to the nonround homogeneous Einstein metric on $S^{7}$

$$
B=\frac{\ln 5}{7}, \quad A=9 \cdot 5^{-10 / 7}\left(1-\frac{r_{h}^{6}}{r^{6}}\right), \quad \delta=0 .
$$

This solution is asymptotically conical so it does not participate in the dynamics of asymptotically flat initial data. It would be interesting to study the evolution of nonasymptotically flat initial data and determine a dynamical role of the solution (12), as well as other known explicit solutions, like, for example, the Spin(7) solution [12]. 
The second difference is the lack of monotonicity of the mass function $m(t, r)$, defined by $A=1-m(t, r) / r^{6}$. From the Hamiltonian constraint (5) we obtain

$$
\begin{aligned}
m^{\prime}= & 3 r^{7}\left(e^{2 \delta} A^{-1} \dot{B}^{2}+A B^{2}\right)+\frac{1}{7} r^{5}\left(42-48 e^{-3 B}\right. \\
& \left.+12 e^{-10 B}-6 e^{4 B}\right) .
\end{aligned}
$$

The potential term on the right hand side of this equation has the local minimum equal to zero at $B=0$, the local maximum at $B=\ln 5 / 7$, and is negative for large positive values of $B$. Thus, the mass density may be locally negative and, indeed, it is easy to construct initial data with large regions of negative mass density. This suggests a possibility of violating the weak cosmic censorship (note that monotonicity of mass is essential in the Dafermos and Holzegel proof [3]). Although our preliminary numerical attempts failed to produce a counterexample, i.e. a generic naked singularity, this problem deserves more systematic investigation. We should stress that despite locally negative mass density the total mass $m(\infty)$ is guaranteed to be nonnegative by the positive mass theorem in higher dimensions [13]. It would be interesting to prove this fact in our model in an elementary manner. In particular, for time- symmetric initial data it follows from Eq. (13) and the requirement of regularity at the origin, $m(0)=0$, that

$$
\begin{aligned}
m(\infty)= & e^{-\int_{0}^{\infty} 3 r B^{12} d r} \int_{0}^{\infty} e^{\int_{0}^{r} 3 \rho B^{12} d \rho}\left(3 r^{7} B^{2}\right. \\
& \left.+\frac{1}{7} r^{5}\left(42-48 e^{-3 B}+12 e^{-10 B}-6 e^{4 B}\right)\right) d r .
\end{aligned}
$$

We challenge the readers to show that this integral is nonnegative for all "reasonable" functions $B(r)$ which vanish at the origin and have compact support (or fall off faster than $r^{-3}$ at infinity). Finally, we remark that there is an analogous problem for the black-hole boundary condition, $m\left(r_{h}\right)=r_{h}^{6}$, where one wants to prove the Penrose inequality $m(\infty) \geq m\left(r_{h}\right)$.

\section{ACKNOWLEDGMENTS}

P. B. is grateful to Gary Gibbons for discussions, remarks and sharing his notes. P. B. acknowledges the hospitality of the Isaac Newton Institute in Cambridge where this work was initiated. This research was supported in part by the Polish Ministry of Science Grant No. 1PO3B01229.
[1] L. Randall and R. Sundrum, Phys. Rev. Lett. 83, 4690 (1999).

[2] P. Bizoń, T. Chmaj, and B. G. Schmidt, Phys. Rev. Lett. 95, 071102 (2005).

[3] M. Dafermos and G. Holzegel, gr-qc/0510051.

[4] A.L. Besse, Einstein Manifolds (Springer, New York, 1987).

[5] M. J. Duff, B.E. W. Nilsson, and C. N. Pope, Phys. Rev. Lett. 50, 2043 (1983).

[6] M. A. Awada, M. J. Duff, and C. N. Pope, Phys. Rev. Lett. 50, 294 (1983).

[7] M. Cvetic, G. W. Gibbons, H. Lu, and C. N. Pope, Nucl.
Phys. B 620, 29 (2002).

[8] G. Gibbons and S. A. Hartnoll, Phys. Rev. D 66, 064024 (2002).

[9] A. Ishibashi and H. Kodama, Prog. Theor. Phys. 110, 901 (2003).

[10] E. W. Leaver, Proc. R. Soc. A 402, 285 (1985).

[11] E. W. Leaver, Phys. Rev. D 41, 2986 (1990).

[12] G.W. Gibbons, D. N. Page, and C.N. Pope, Commun. Math. Phys. 127, 529 (1990).

[13] J. Lohkamp, Math. Ann. 313, 385 (1999).

[14] K. D. Kokkotas and B. G. Schmidt, Living Rev. Relativity 2, 2 (1999). 